\title{
Similarity Solutions of Strong Shock Waves for Isothermal Flow in an Ideal Gas
}

\author{
Astha Chauhan \\ Department of Applied Science and Engineering \\ Indian Institute of Technology Roorkee, Roorkee, Uttarakhand, India \\ Corresponding author: asthaiitr1@gmail.com \\ Rajan Arora \\ Department of Applied Science and Engineering \\ Indian Institute of Technology Roorkee, Roorkee, Uttarakhand, India \\ E-mail: rajan_a100@yahoo.com
}

(Received March 16, 2018; Accepted July 15, 2019)

\begin{abstract}
Self-similar solutions of the system of non-linear partial differential equations are obtained using the Lie group of invariance technique. The system of equations governs the one dimensional and unsteady motion for the isothermal flow of an ideal gas. The medium has been taken the uniform. From the expressions of infinitesimal generators involving arbitrary constants, different cases arise as per the choice of the arbitrary constants. In this paper, the case of a collapse of an implosion of a cylindrical shock wave is shown in detail along with the comparison between the similarity exponent obtained by Guderley's method and by Crammer's rule. Also, the effects of the adiabatic index and the ambient density exponent on the flow variables are illustrated through the figures. The flow variables are computed behind the leading shock and are shown graphically.
\end{abstract}

Keywords- Lie group, Shock waves, Rankine-Hugoniot conditions, Similarity solutions, Ideal gas.

\section{Introduction}

Study of shock waves propagation is always a topic of great interest for researchers. Because this phenomenon is well- marked in the disassembled nature of the absorption of energy. Shock waves form when the velocity of the object becomes higher than the velocity of sound. The equations of gas dynamics govern the continuous flow behind the shock, which is solved subject to the Rankine-Hugoniot conditions. These equations are present in the form of non-linear partial differential equations. There are many forms of self-similar solutions describing the implosions. A theoretical study on the imploding shock wave was first performed by Guderley (1942). His work was based on an analytical study. After a decade, Perry and Kantrowitz (1951) followed his work by producing shocks experimentally. Van Dyke and Guttman (1982), and Hafner (1988), used another approach to study the imploding shock waves in a perfect gas. Many research groups have worked afterward on the topic to better understand the dynamics of shock waves. Among the recent research on the topic, we wish to mention the work of Hydon (2000), Singh et al. (2010), and Nath and Singh (2017).

The Lie group method of invariance is widely used to compute the invariant solutions for the problem of gas dynamics involving shock phenomena. This method helps us to simplify the complicated problem involving any physical phenomena into the solvable mathematical system of equations. The basic idea of Lie group method for solving the physical problem, which has been formulated in the form of mathematical equations, can be found in the work presented by 
International Journal of Mathematical, Engineering and Management Sciences

Vol. 4, No. 5, 1094-1107, 2019

https://dx.doi.org/10.33889/IJMEMS.2019.4.5-087

Logan (1987), Bluman and Kumei (1989), Sharma and Radha (1995), Hydon (2000), Arora and Chauhan (2019) used this method in their work to obtain the exact similarity solutions of KDVBurgers equations. In 2013, Arora et al. (2013) used the Lie group method to obtain the high accuracy results for the implosion problem in a non-ideal relaxing gas. Arora and Sharma (2016) studied the propagation of cylindrical shock waves in magnetogasdynamics with a radiation effect. A particular solution is obtained by them for the problem in an ideal gas.

Generally, it is very difficult to obtain the solution of the system hyperbolic PDEs. Therefore, we seek similarity solutions subject to the shock conditions along which the system of PDEs is transformed into the system of ODEs. For determining the similarity solutions, there are two methods to obtain the value of similarity exponent. For the first method, similarity exponent is obtained by either conservation laws or by dimensional considerations and in the second method; the similarity exponent is determined by integrating the reduced ODEs (Zeldovich and Raizer, 1965). In the present work, we have used the second method to obtain the values of similarity exponent.

When effects of radiation are already present implicitly, then the assumption for the isothermal flow becomes physically realistic. As the shock propagates, the temperature (behind the shock) starts increasing and becomes very high. Due to this, the temperature gradient approaches to zero. Hence, the flow becomes isothermal. Vishwakarma and Nath (2007) have determined the similarity solution of shock wave in a non-ideal gas for the isothermal flow where density varies exponentially. Recently, Chauhan and Arora (2019) studied the strong shock problem in a dusty gas for isothermal thermal flow with an added magnetic field. They have also shown the comparison between isothermal and adiabatic flows. The shock wave propagation influenced by the magnetic field is very interesting, especially in the coronal heating problem. This problem is related to the temperature of the sun as its corona has a much higher temperature than that of its surface. Therefore, the effect of the magnetic field on a characteristic wave or shock wave is important to know the physics of the phenomena as well. In the past few years, many researchers have worked on the topic to better understand the physics of the involved physical phenomena (Singh and Vishwarkarma, 1983; Singh et al., 2010; Chauhan et al., 2019).

In this paper, we have applied Lie group method to find the self-similar solutions of the equations which govern the one-dimensional unsteady cylindrically symmetric motion for the isothermal flow of an ideal gas, with the effect of the axial magnetic field, and obtained infinitesimal generators. The main advantage of this method is to reduce the number of independent variables by one. The values of the similarity exponent are obtained for two different values of the adiabatic index. The patterns of flow variables just behind the shock are obtained by performing numerical computations using the software Mathematica. The rest of the paper is summarized as follows: Section 1 contains a brief introduction to the topic and historical background of earlier studies. Section 2 presents the fundamental equations of motions governing the conservation laws. Rankine-Hugoniot (RH) jump conditions are also presented in this section. In Section 3, the Lie group of invariance technique is used to obtain the infinitesimal group of generators of the given system of equations. With the help of infinitesimals, the given system of partial differential equations (PDEs) is reduced into the system of ordinary differential equations (ODEs). In Section 4 , a detailed study is shown about the collapse of an imploding shock in the neighborhood of the implosion. Section 5 presents the results and discussion about the study. In the disturbed region, the effects of the ambient density exponent and the adiabatic index on the flow parameters are also discussed in this section. Section 6 is the conclusion. 
International Journal of Mathematical, Engineering and Management Sciences

Vol. 4, No. 5, 1094-1107, 2019

https://dx.doi.org/10.33889/IJMEMS.2019.4.5-087

\section{Equations of Motions and Boundary Conditions}

The basic equations of motion, which describe the one-dimensional unsteady and cylindrically symmetric motion for isothermal flow of a perfectly conducting gas, with the effect of the axial magnetic field, are given as follows (Singh and Vishwakarma, 1983):

$$
\begin{aligned}
& \frac{\partial \rho}{\partial t}+u \frac{\partial \rho}{\partial r}+\rho \frac{\partial u}{\partial r}+\frac{\rho u}{r}=0, \\
& \frac{\partial u}{\partial t}+u \frac{\partial u}{\partial r}+\frac{1}{\rho}\left(\frac{\partial \rho}{\partial r}+h \frac{\partial h}{\partial r}\right)=0 \\
& \frac{\partial h}{\partial t}+u \frac{\partial h}{\partial r}+h \frac{\partial u}{\partial r}+\frac{h u}{r}=0 \\
& \frac{\partial p}{\partial r}-\frac{p}{\rho} \frac{\partial \rho}{\partial r}=0 .
\end{aligned}
$$

Here $\rho, u, p$, and $h$ represent the density, velocity, pressure and magnetic field, respectively. The effects of heat conduction and viscosity are not considered, and the electrical conductivity of the gas is supposed to be infinite. The equation of state is given by

$p=\rho R T$

Here $R$ is the specific gas constant and $T$ is the temperature. Let $r=\hat{R}(t)$ denotes the shock wave front motion, propagating into a medium specified with the help of following equations:

$u_{0}=0, \quad p_{0}=a_{1}$ (constant $), \quad \rho_{0}=\rho_{0}(r), \quad h_{0}=h_{0}(r)$,

where the suffix 0 indicates the flow in undisturbed medium and $a_{1}$ is a fix constant. Let $V$ be the velocity of the shock wave, then the jump conditions behind a strong shock for the given problem may be written as (Whitham, 2011):

$u=\frac{2 V}{\gamma+1}, \quad \rho=\frac{\gamma+1}{\gamma-1} \rho_{0}, \quad p=\frac{2 V^{2} \rho_{0}}{\gamma+1}, \quad h=\frac{\gamma+1}{\gamma-1} h_{0}$,

where $\gamma$ is the adiabatic index.

\section{Similarity Analysis}

For obtaining the similarity solutions, we find a set of an infinitesimal group of transformations (Arora, 2005) in such a way that the equations (1) -(4) remain invariant with respect to this group of transformations and yield the similarity solutions. Let us consider the infinitesimal group of transformations as follows:

$$
\begin{aligned}
& r^{*}=r+\epsilon \chi(\rho, u, h, p, r, t), \quad t^{*}=t+\epsilon \psi(\rho, u, h, p, r, t), \\
& u^{*}=u+\epsilon U(\rho, u, h, p, r, t), \quad \rho^{*}=\rho+\epsilon S(\rho, u, h, p, r, t), \\
& p^{*}=p+\epsilon P(\rho, u, h, p, r, t), \quad h^{*}=h+\epsilon E(\rho, u, h, p, r, t),
\end{aligned}
$$

where $\chi, \psi, U, S, P$ and $E$ are the infinitesimal generators such that the equations (1)-(4) along with the shock conditions (7) remain unchanged under the above infinitesimal group (8), $\epsilon$ is very small quantity such that we can neglect its second and higher power terms. This group decreases 
International Journal of Mathematical, Engineering and Management Sciences

Vol. 4, No. 5, 1094-1107, 2019

https://dx.doi.org/10.33889/IJMEMS.2019.4.5-087

the number of independent variables of the given system by one and thus, we obtain the system of first order nonlinear ordinary differential equations.

The notations used are as follows:

$u_{1}=\rho, u_{2}=u, u_{3}=p, u_{4}=h, x_{1}=t, x_{2}=r$ and $p_{j}^{i}=\frac{\partial u_{i}}{\partial x_{j}}$, where $i=1,2,3,4$ and $j=$ 1,2 .

The system of basic equations (1)-(4), which can be expressed as

$F_{s}\left(x_{j}, u_{i}, p_{j}^{i}\right)=0, \quad s=1,2,3,4$

is said to be invariant with respect to the Lie group (8) iff

$L F_{s}=\alpha_{s r} F_{r}$, where $s=1,2,3,4$ and $r=1,2,3,4$

where L represents the Lie derivative given as:

$L=\xi_{x}^{j} \frac{\partial}{\partial x_{j}}+\xi_{u}^{j} \frac{\partial}{\partial u_{i}}+\xi_{p_{j}}^{j} \frac{\partial}{\partial p_{j}^{i}}$

with $\xi_{x}^{1}=\psi, \xi_{x}^{2}=\chi, \xi_{u}^{1}=S, \xi_{u}^{2}=U, \xi_{u}^{3}=P, \xi_{u}^{4}=E$ and

$\xi_{p_{j}}^{j}=\frac{\partial \xi_{u}^{i}}{\partial x_{j}}+\frac{\partial \xi_{u}^{i}}{\partial u_{k}} p_{l}^{i}-\frac{\partial \xi_{x}^{l}}{\partial x_{j}}-\frac{\partial \xi_{x}^{l}}{\partial u_{n}} p_{l}^{i} p_{j}^{n}$

where $l=1,2, j=1,2, n=1,2,3,4$ and $k=1,2,3,4$.

So, the Eq. (9) can be written as

$\xi_{x}^{j} \frac{\partial F_{s}}{\partial x_{j}}+\xi_{u}^{j} \frac{\partial F_{s}}{\partial u_{i}}+\xi_{p_{j}}^{j} \frac{\partial F_{s}}{\partial p_{j}^{i}}=\alpha_{s r} F_{r}$, where $r, s=1,2,3,4$

Substitution of $\xi_{p_{j}}^{j}$ from Eq. (10) into Eq. (11) yields a polynomial equation in $p_{j}^{i}$. Equating to zero the coefficients of $p_{j}^{i} p_{l}^{n}$ on both sides of Eq. (11), we obtain a system of non-linear PDEs in the terms of the infinitesimals generators $\chi, \psi, U, S, P$ and $E$. After solving these systems of PDEs, we get the following set of infinitesimals:

$\chi=\left(\alpha_{22}+2 a\right) r, \quad \psi=a t+b, \quad S=\left(\alpha_{11}+a\right) \rho$,

$U=\left(\alpha_{22}+a\right) u, \quad P=\left(\alpha_{11}+2 \alpha_{22}+3 a\right) p, \quad E=\frac{1}{2}\left(\alpha_{11}+2 \alpha_{22}+3 a\right) h$,

where $\alpha_{11}, \alpha_{22}, a$ and $b$ are the arbitrary constants.

Case 1: Considering $a \neq 0$ and $\left(\alpha_{22}+2 a\right)=0$, the change variables $\tilde{r}$ and $\tilde{t}$ are defined as 
International Journal of Mathematical, Engineering and Management Sciences

Vol. 4, No. 5, 1094-1107, 2019

https://dx.doi.org/10.33889/IJMEMS.2019.4.5-087

$\tilde{r}=r, \tilde{t}=t+\frac{b}{a}$.

We find that Eq. (1) -(12) remain invariant under the above transformations (13). Therefore, using (13) and suppressing the asterisk sign, we have

$\chi=\left(\alpha_{22}+2 a\right) r, \quad \psi=a, \quad S=\left(\alpha_{11}+a\right) \rho$,

$U=\left(\alpha_{22}+a\right) u, \quad P=\left(\alpha_{11}+2 \alpha_{22}+3 a\right) p, \quad E=\frac{1}{2}\left(\alpha_{11}+2 \alpha_{22}+3 a\right) h$.

The invariant surface conditions given in (Logan, 1987) are as follows:

$\psi \rho_{t}+\chi \rho_{r}=S, \quad \psi u_{t}+\chi u_{r}=U$,

$\psi p_{t}+\chi p_{r}=P, \quad \psi h_{t}+\chi h_{r}=E$.

After performing integration of the equations in (15) together with equations (14), the flow variables are obtained in the following forms

$\begin{aligned} \rho & =t^{\left(1+\frac{\alpha_{11}}{a}\right)} \bar{S}(\xi), & u & =t^{(\delta-1)} \bar{U}(\xi), \\ p & =t^{\left(2 \delta-11+\frac{\alpha_{11}}{a}\right)} \bar{P}(\xi), & h & =t^{\frac{1}{2}\left(2 \delta-11+\frac{\alpha_{11}}{a}\right)} E(\xi),\end{aligned}$

where

$\delta=\frac{\alpha_{22}+2 a}{a}$

and $\bar{S}, \bar{U}, \bar{P}$ and $\bar{E}$ are dependent only on the similarity variable $\xi$, which is obtained as

$\xi=\frac{r}{t^{\delta}}$

Since the shock is a similarity curve. Therefore, it may be normalized at $\xi=1$. So, the expression for the shock path $\hat{R}(t)$ and the shock velocity $V(t)$ are given as

$\hat{R}(t)=t^{\delta}, \quad V=\frac{\delta \hat{R}}{t}$.

At the shock, $\xi=1$, we have the expressions for the flow variables given as follows:

$\begin{array}{ll}\left.\rho\right|_{\xi=1}=t^{\left(1+\frac{\alpha_{11}}{a}\right) \bar{S}(1),} & \left.u\right|_{\xi=1}=t^{(\delta-1) \bar{U}(1)} \\ \left.p\right|_{\xi=1}=t^{\left(2 \delta-1+\frac{\alpha_{11}}{a}\right)} \bar{P}(1), & \left.h\right|_{\xi=1}=t^{\frac{1}{2}\left(2 \delta-1+\frac{\alpha_{11}}{a}\right)} \bar{E}(1) .\end{array}$

From the invariance conditions, the following forms of $\rho_{0}(r)$ and $h_{0}(r)$ are obtained as follows:

$\rho_{0}(r)=\rho_{c} r^{\theta}, \quad h_{0}(r)=h_{c} r^{\lambda}$ 
International Journal of Mathematical, Engineering and Management Sciences

Vol. 4, No. 5, 1094-1107, 2019

https://dx.doi.org/10.33889/IJMEMS.2019.4.5-087

and at the shock $\xi=1$, the boundary conditions are obtained as follows:

$$
\begin{aligned}
& \bar{U}(1)=\frac{2}{\gamma+1}, \quad \bar{S}(1)=\frac{\gamma+1}{\gamma-2} \rho_{c}, \\
& \bar{P}(1)=\frac{2}{\gamma+1} \delta^{2} \rho_{c}, \quad \bar{E}(1)=\frac{\gamma+1}{M_{A}(\gamma+1)} \rho_{c}^{1 / 2} \delta,
\end{aligned}
$$

together with

$$
\theta=\frac{\alpha_{11}+a}{\alpha_{22}+2 a}, \quad \lambda=\frac{\alpha_{11}+2 \alpha_{22}+3 a}{2\left(\alpha_{22}+2 a\right)}
$$

where $\rho_{c}$ and $\theta$ are the constants associated with the medium and $M_{A}=\frac{V \rho_{0}^{1 / 2}}{h_{0}}$ is the Alfven Mach number.

Using Eq. (18), (19) and (21), we obtain the Eq. (16) in the following forms:

$$
\begin{aligned}
\rho & =\rho_{0}(\hat{R}(t)) \grave{S}(\xi), & & u=V \grave{U}(\xi), \\
p & =p_{0}(\hat{R}(t)) V^{2} \grave{P}(\xi), & h & =\rho_{0}^{1 / 2} V \grave{E}(\xi),
\end{aligned}
$$

where $\grave{S}(\xi)=\frac{\bar{S}(\xi)}{\rho_{c}}, \grave{U}(\xi)=\frac{\bar{U}(\xi)}{\delta}, \grave{P}(\xi)=\frac{\bar{P}(\xi)}{\delta^{2} \rho_{c}}$ and $\grave{E}(\xi)=\frac{\bar{E}(\xi)}{\delta \rho_{c}^{1 / 2}}$.

Substituting Eq. (24) in the equations (1) -(4) and using Eq. (16), (19) and (24) and dropping the sign (") the transformed system of ODEs is obtained as

$$
\begin{aligned}
& S \theta+(U-\xi) S^{\prime}+S U^{\prime}+\frac{U}{\xi} S=0, \\
& (U-\xi) S U^{\prime}+\frac{(\delta-1)}{\delta} S U+P^{\prime}+E E^{\prime}=0, \\
& (U-\xi) E^{\prime}+U^{\prime} E+\left[\frac{U}{\xi}+\frac{\delta-1}{\delta}+\frac{\theta}{2}\right] E=0, \\
& P^{\prime} S-P S^{\prime}=0 .
\end{aligned}
$$

For the strong shocks, the R-H conditions are given by

$$
\begin{aligned}
& U(1)=\frac{2}{\gamma+1}, \quad S(1)=\frac{\gamma+1}{\gamma-2} \\
& P(1)=\frac{2}{\gamma+1}, \quad E(1)=\frac{\gamma+1}{M_{A}(\gamma+1)} .
\end{aligned}
$$

Case 2: Let us consider the case when $a=0$ and $\alpha_{22}=0$. Therefore, a new set of infinitesimals is obtained as 
International Journal of Mathematical, Engineering and Management Sciences

Vol. 4, No. 5, 1094-1107, 2019

https://dx.doi.org/10.33889/IJMEMS.2019.4.5-087

$\chi=\left(\alpha_{22}\right) r, \quad \psi=b, \quad S=\left(\alpha_{11}\right) \rho$,

$U=\left(\alpha_{22}\right) u, \quad P=\left(\alpha_{11}+2 \alpha_{22}\right) p, \quad E=\frac{1}{2}\left(\alpha_{11}+2 \alpha_{22}\right) h$.

The equations in (15) together with equations (27), after performing integration, yield the flow variables in the following form:

$\begin{aligned} \rho & =e^{\frac{\alpha_{11}}{a} t} \bar{S}(\xi), & u & =e^{\delta t} \bar{U}(\xi), \\ p & =e^{\left(2 \delta+\frac{\alpha_{11}}{a}\right) t} \bar{P}(\xi), & h & =e^{\frac{1}{2}\left(2 \delta+\frac{\alpha_{11}}{a}\right) t} \bar{E}(1),\end{aligned}$

where

$\delta=\frac{\alpha_{22}}{a}$

The expression for the similarity variable $\xi$, shock path $\hat{R}(t)$ and the shock velocity $V(t)$ are given as

$\xi=e^{-\delta t}, \quad \hat{R}(t)=e^{\delta t}, \quad V=\delta e^{\delta t}$.

Therefore, the flow variables $\rho, u, p, h, \rho_{0}(r)$ and $h_{0}(r)$ are obtained in the following forms:

$\rho=\rho_{0}(\hat{R}(t)) \grave{S}(\xi), \quad u=V \grave{U}(\xi)$

$p=p_{0}(\hat{R}(t)) V^{2} \grave{P}(\xi), \quad h=\rho_{0}^{\frac{1}{2}} V \grave{E}(\xi)$,

$\rho_{0}(r)=\rho_{c} r^{\theta}, \quad h_{0}(r)=h_{c} r^{\lambda}$

$\theta=\frac{\alpha_{11}}{\alpha_{22}}, \quad \lambda=\frac{\alpha_{11}+2 \alpha_{22}}{\alpha_{22}}$

where $\theta$ and $\rho_{c}$ are the constants associated with the medium. Also, $\grave{S}(\xi)=\frac{\bar{S}(\xi)}{\rho_{c}}, \grave{U}(\xi)=\frac{\bar{U}(\xi)}{\delta}$, $\grave{P}(\xi)=\frac{\bar{P}(\xi)}{\delta^{2} \rho_{c}}$ and $\grave{E}(\xi)=\frac{\bar{E}(\xi)}{\delta \rho_{c}^{1 / 2}}$

From Eq. (29), we see that the shock path is exponentially varying. Substituting Eq. (30) in the equations (1)-(4), we obtain the following system of ODEs after suppressing the asterisk signs

$$
\begin{aligned}
& S \theta+(U-\xi) S^{\prime}+S U^{\prime}+\frac{U}{\xi} S=0, \\
& (U-\xi) S U^{\prime}+S U+P^{\prime}+E E^{\prime}=0,
\end{aligned}
$$


International Journal of Mathematical, Engineering and Management Sciences

Vol. 4, No. 5, 1094-1107, 2019

https://dx.doi.org/10.33889/IJMEMS.2019.4.5-087

$(U-\xi) E^{\prime}+\left(\frac{U}{\xi}+U^{\prime}\right) E+\left(1+\frac{\theta}{2}\right)=0$,

$P^{\prime} S-P S^{\prime}=0$.

For the strong shocks, the R-H conditions are given by

$$
\begin{array}{ll}
U(1)=\frac{2}{\gamma+1}, & S(1)=\frac{\gamma+1}{\gamma-2}, \\
P(1)=\frac{2}{\gamma+1}, & E(1)=\frac{\gamma+1}{M_{A}(\gamma+1)} .
\end{array}
$$

The system (32) together with the jump conditions (33) may be solved for the shock of infinite strength.

\section{Imploding Shocks}

In this section, we consider an imploding shock wave in the implosion's neighborhood, when an imploding shock is about to collapse at the center or axis, we assume the origin of time $t$ at which the shock reaches the center to be the instant, thereby $t \leq 0$ in (25). Thus, we slightly modify the definition of $\xi$, by using the following transformations:

$\xi=\frac{r}{A(-t)^{\delta}}, \quad \hat{R}(t)=A(-t)^{\delta}$.

Therefore, for the flow variables, the intervals $-\infty<t \leq 0, \hat{R} \leq r<\infty$ and $1 \leq \xi<\infty$. At any finite radius $r$, the gas density, pressure, velocity and the speed of sound are bounded, but for $t=$ 0 and finite $r, \xi=\infty$ (i.e. instant of collapse). For the variables $\rho, u, p$ and $h$ to be bounded, the boundary conditions at the, $\xi=\infty$ is as follows:

$U(\infty)=0, \quad \frac{E(\infty)}{S(\infty)}=0, \quad \frac{P(\infty)}{S(\infty)}=0$

Table 1. Values of similarity exponent $\delta$ for different values of $\gamma$ and ambient density exponent $\theta$

\begin{tabular}{|c|c|c|c|c|}
\hline$\gamma$ & $\theta$ & Computed $\boldsymbol{\delta}$ & Guderley's $\boldsymbol{O}$ & \% Error \\
\hline & 0.1 & 0.7063 & 0.7062 & 0.01 \\
\hline & 0.5 & 0.6237 & 0.5875 & 3.62 \\
\hline & 1.0 & 0.5375 & 0.5250 & 0.25 \\
\hline & 1.5 & 0.4710 & 0.4750 & 5.01 \\
\hline & 2.0 & 0.4000 & 0.4501 & 0.50 \\
\hline & 0.1 & 0.6800 & 0.6750 & 4.37 \\
\hline & 0.5 & 0.5812 & 0.5375 & 6.01 \\
\hline & 1.0 & 0.4400 & 0.5001 & 0.07 \\
\hline
\end{tabular}


International Journal of Mathematical, Engineering and Management Sciences

Vol. 4, No. 5, 1094-1107, 2019

https://dx.doi.org/10.33889/IJMEMS.2019.4.5-087

The system (25) can be written in the form of matrix as:

$B W^{\prime}=C$,

where $W=(S, U, P, E)^{t r}$. The matrix $B$ and the column vector $C$ can be obtained with the help of the system (25). It may be noticed that there is an unknown value $\delta$ in the system (25), which cannot be obtained from the dimensional considerations; it is computed for a system of ODEs from a non-linear eigenvalue problem. Now, the system (36) can be solved for the variables $U$ ', $S^{\prime}, P^{\prime}$ and $E^{\prime}$ using the Cramer's rule in the following manner:

$S^{\prime}=\frac{\Delta_{2}}{\Delta}, \quad U^{\prime}=\frac{\Delta_{1}}{\Delta}, \quad P^{\prime}=\frac{\Delta_{3}}{\Delta}, \quad E^{\prime}=\frac{\Delta_{4}}{\Delta}$,

where $\Delta$ represents matrix $B$ 's determinant and is given by

$\Delta=S(U-\xi)\left[(U-\xi)^{2}-\frac{\left(E^{2}+P\right)}{S}\right]$.

Also, $\Delta_{k}(k=1,2,3,4)$ represent the matrix $B$ 's determinants, which can be obtained from matrix $A$ by interchanging its $k^{\text {th }}$ column with column vector $C$. In the interval $[1, \infty)$, it may be noticed that $U<\xi$. So, $\Delta>0$ at $\xi=1$ and $\Delta<0$ at $\xi=\infty$, which implies that there exist a $\xi \in[1, \infty)$ at which $\Delta$ becomes zero. Therefore, the solutions of the system (37) become singular. Hence for obtaining a solution of (36), we choose the value of the exponent $\delta$ in such a manner that $\Delta$ becomes zero only at the points, where the determinants $\Delta_{1}, \Delta_{2}, \Delta_{3}$, and $\Delta_{4}$ also becomes zero simultaneously. Introducing the variable $Z$ to obtain the correct value of exponent $\delta$ as follows:

$Z(\xi)=\left[(U-\xi)^{2}-\frac{\left(E^{2}+P\right)}{S}\right]$

whose first derivative, in view of (37) is as follows

$\frac{d Z}{d \xi}=\left[2(U-\xi)\left(\Delta_{1}-\Delta\right)-\frac{\left(\Delta_{3}+2 E \Delta_{4}\right)}{S}+\frac{\left(E^{2}+P\right)}{S^{2}} \Delta_{2}\right] / \Delta$.

Therefore, the equations (37), with the help of Eq. (40), become

$\frac{d U}{d Z}=\frac{\Delta_{1}}{\Delta_{5}}, \frac{d S}{d Z}=\frac{\Delta_{2}}{\Delta_{5}}, \quad \frac{d P}{d Z}=\frac{\Delta_{3}}{\Delta_{5}}, \quad \frac{d E}{d Z}=\frac{\Delta_{4}}{\Delta_{5}}$,

where

$\Delta_{5}=\left[2(U-\xi)\left(\Delta_{1}-\Delta\right)-\frac{\left(\Delta_{3}+2 E \Delta_{4}\right)}{S}+\frac{\left(E^{2}+P\right)}{S^{2}} \Delta_{2}\right]$, with $\xi=U+\left(Z+\frac{P+E^{2}}{S}\right)^{1 / 2}$.

\section{Results and Discussion}

On integrating Eqs. (41) for $1 \leq \xi<\infty$ by assigning some value to $\delta$, we obtain the values of $S, U$, $P, E$ and at $Z=0$. The computed values of $\delta$ are listed in Table 1 for different values of similarity exponent $\theta$ and $\gamma$, which also depict the comparison with the corresponding values of $\delta$ obtained by Guderley's method. From Table 1, we observe that as the value of $\gamma$ and $\theta$ increase, the value of similarity exponent $\delta$ decreases $M_{A}{ }^{-2}$ and $\gamma$ are taken as $M_{A}{ }^{-2}=0.02, \gamma=7 / 5,5 / 3$. The magnetic 
International Journal of Mathematical, Engineering and Management Sciences

Vol. 4, No. 5, 1094-1107, 2019

https://dx.doi.org/10.33889/IJMEMS.2019.4.5-087

field effects on the flow are evincive when $M_{A}^{-2} \geq 0.01$ (Rosenau and Frankenthal, 1976). It can be easily observed that the computed results are in good agreement with those obtained from Guderley's method.

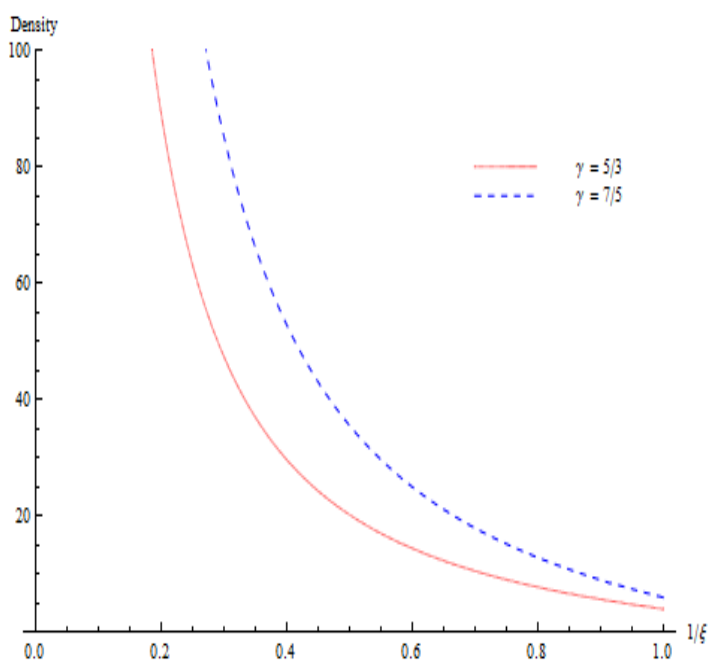

(i)

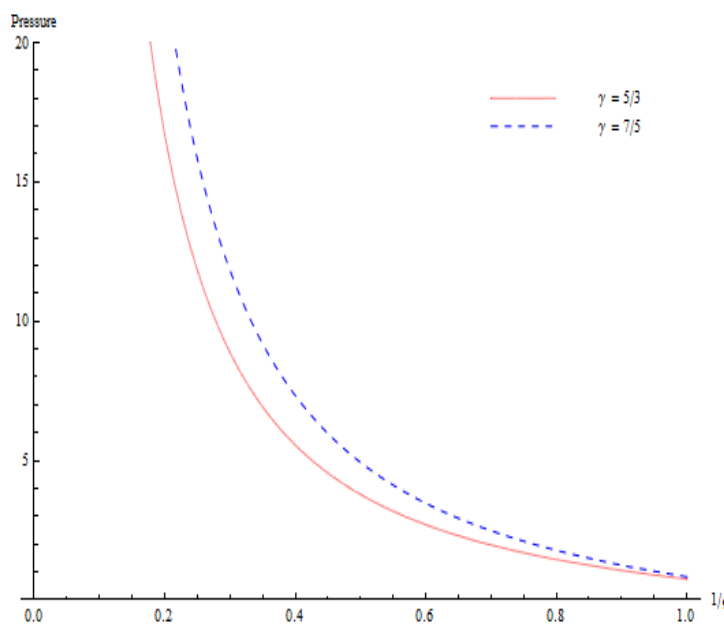

(iii)

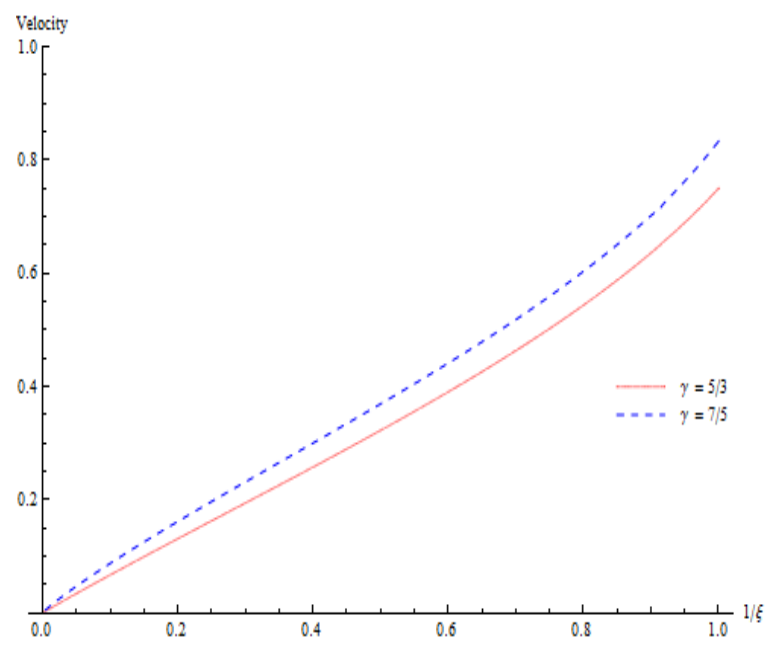

(ii)

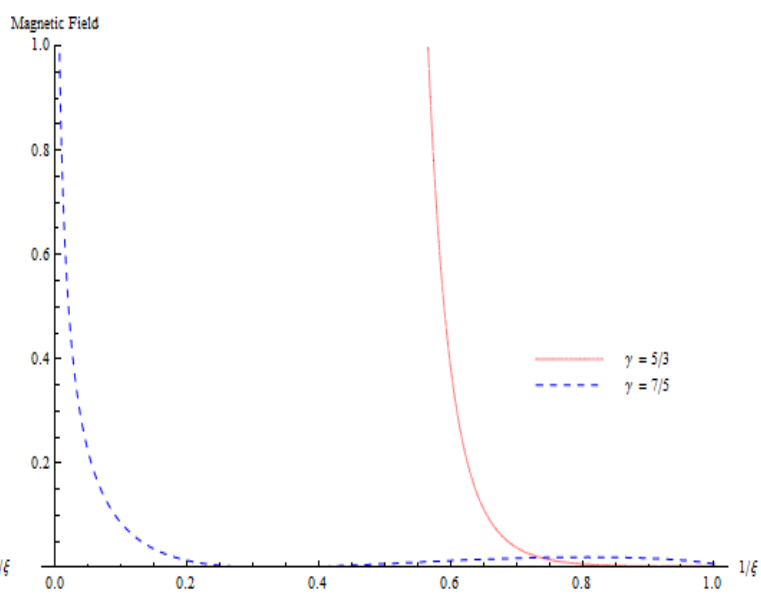

(iv)

Figure 1. Flow profiles of (i) density, (ii) velocity, (iii) pressure and (iv) magnetic field for $\gamma=7 / 5,5 / 3$

The expressions of the flow variables, obtained from Eq. (37), where the shock wave collapse at $t$ $=0, X=0(t=0, r \neq 0, \xi=\infty)$, are given as

$U \sim \xi^{(\delta-1) / \delta}, \quad S \sim K, \quad P \sim \xi^{2(\delta-1) / \delta}, E \sim \xi^{2(\delta-1) / \delta}$ as $\xi \rightarrow \infty$

where $K$ is a constant. 
International Journal of Mathematical, Engineering and Management Sciences

Vol. 4, No. 5, 1094-1107, 2019

https://dx.doi.org/10.33889/IJMEMS.2019.4.5-087

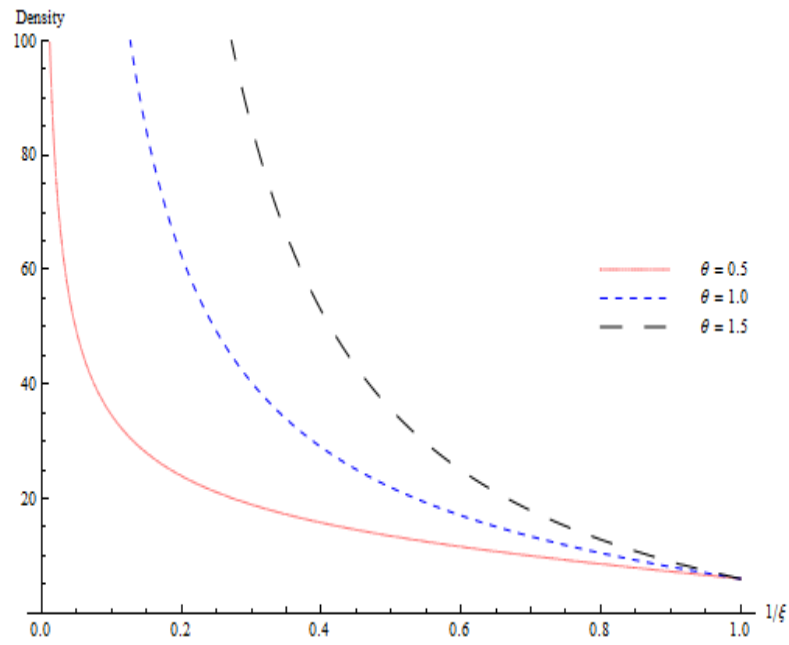

(i)

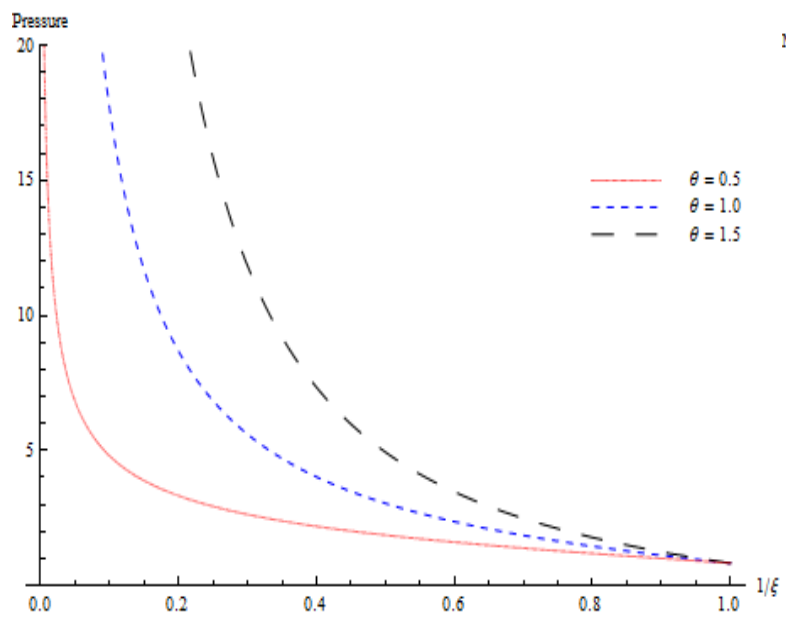

(iii)

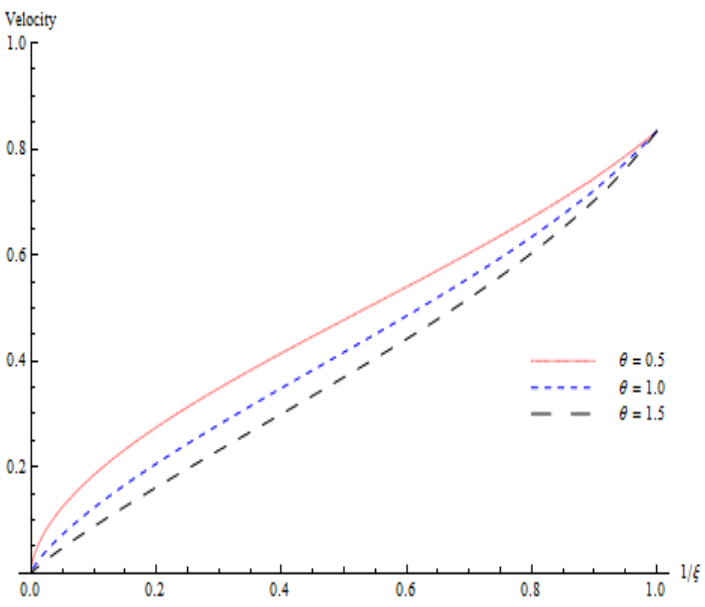

(ii)

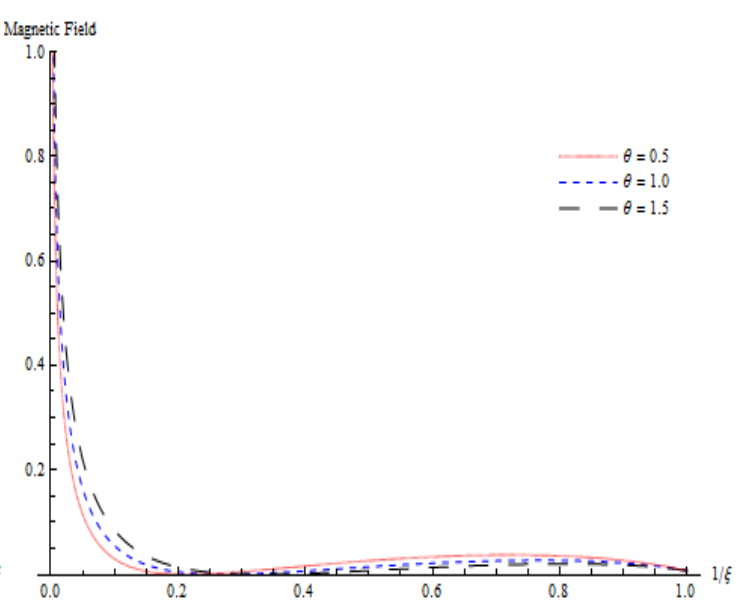

(iv)

Figure 2. Flow profiles of (i) density, (ii) velocity, (iii) pressure and (iv) magnetic field for $\gamma=7 / 5$ and $\theta=0.5,1.0,1.5$

From the expressions in (42), we observe that the velocity $U$ and the pressure $P$ tend to zero as $\xi<\infty$, at the instant collapse due to the value of $\delta$ smaller than unity, while the density $S$ remains bounded thereat. The system (37) is integrated into the range $1 \leq \xi<\infty$ and the profiles of the variables $\rho, u, p$ and $h$ are illustrated in Figures 1-3. The numerical solutions of flow variables in the neighborhood of implosion are consistent with the results, which are asymptotic in nature.

When we go towards the center of collapse, from the flow profiles shown in Figures 1-3, we observe that the density, pressure and magnetic field increase while the velocity decreases behind the shock. The gas-particle, which passes through the shock leads to the compression of shock, which increases the density, pressure and magnetic field behind the shock and it may also be attributed to the area contraction or the convergence of the shock wave. 
International Journal of Mathematical, Engineering and Management Sciences

Vol. 4, No. 5, 1094-1107, 2019

https://dx.doi.org/10.33889/IJMEMS.2019.4.5-087

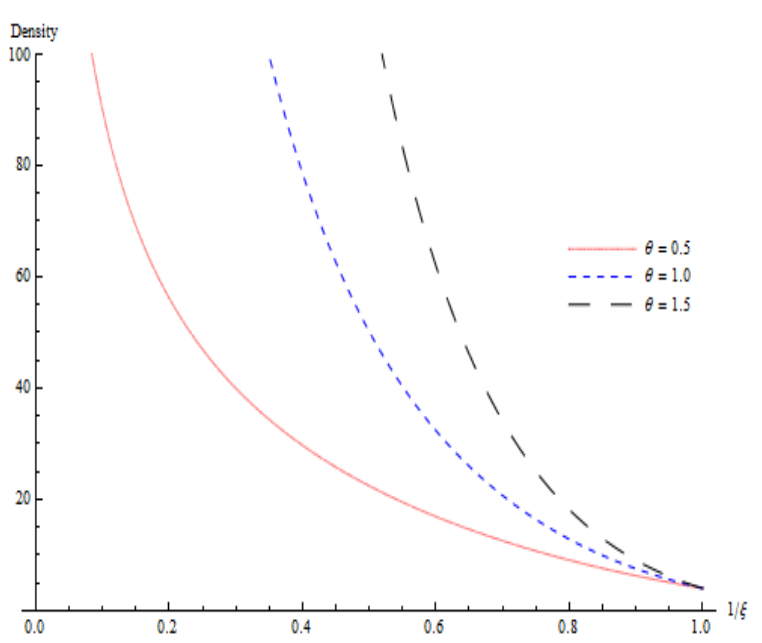

(i)

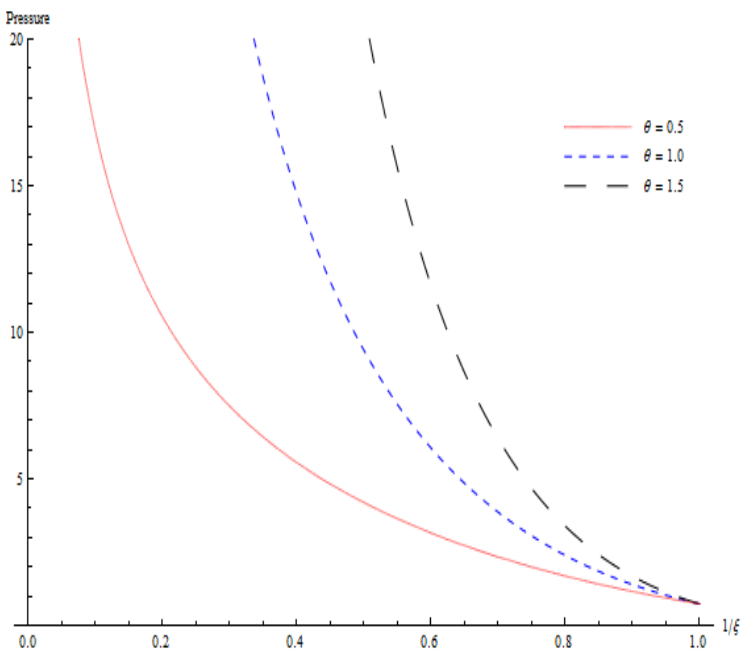

(iii)

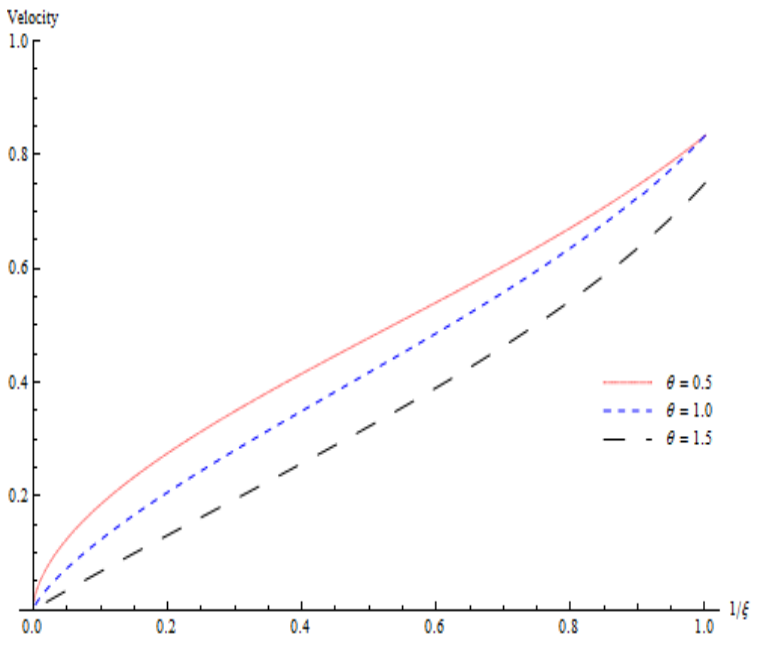

(ii)

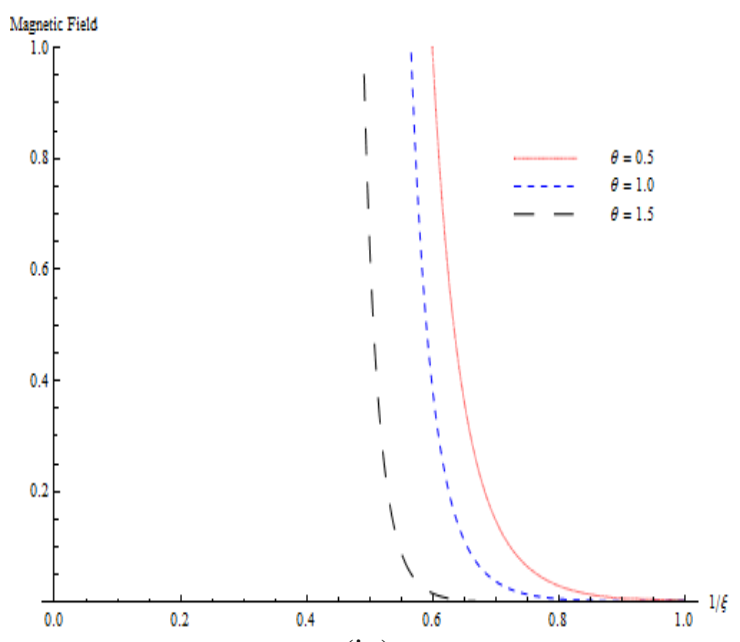

(iv)

Figure 3. Flow profiles of (i) density, (ii) velocity, (iii) pressure and (iv) magnetic field for $\gamma=5 / 3$ and $\theta=0.5,1.0,1.5$

From Figures 1-3, it can be easily observed that the profiles of all the flow variables have monotonic behavior. From Figure 1(i-iv), it is observed that as the value of $\gamma$ increases, all the flow variables decrease. As we increase the value of ambient density exponent $\theta$, the density and the pressure increase while velocity decreases (see Figures 2 and 3). Also, the flow patterns of density and pressure are the same for $\gamma=7 / 5,5 / 3$ (Eq. (4) shows the same result). Further, it may be noted that the Crammer's rule produces a very good approximation for similarity exponents in an ideal medium (see Table 1). 
International Journal of Mathematical, Engineering and Management Sciences

Vol. 4, No. 5, 1094-1107, 2019

https://dx.doi.org/10.33889/IJMEMS.2019.4.5-087

\section{Conclusion}

In this work, the Lie group method is adapted to solve the system of equations describing the unsteady and isothermal flow of an ideal gas. The solutions are obtained for the flow of a perfectly conducting gas behind a strong shock. By using the invariance condition of the Lie group method, we have obtained the set of infinitesimal generators given by (12). A system (25) of ordinary differential equations is obtained with the help of infinitesimal generators and for numerical results, the system (25) is integrated by $4^{\text {th }}$ order Runge-Kutta method for different values of $\gamma$ and exponent ambient density $\theta$ to obtain the suitable values of similarity exponent $\delta$. Also, we have obtained the values of $\delta$ by Guderley's method and shown the comparison between the Guderley's $\delta$ and computed $\delta$ by Crammer's rule for $\gamma=7 / 5,5 / 3$ in Table 1 . The flow profiles of the variables $\rho, u, p$, and $h$ are presented in Figures 1-3. Monotonic behavior is present in the profiles of all the flow variables.

\section{Conflict of Interest}

The authors declare that they have no conflict of interest.

\section{Acknowledgements}

One of the authors, Astha Chauhan is thankful to the 'University Grant Commission, New Delhi for providing research grant (Grant no. 2121440656, Ref. No. 21/12/2014 (ii) EU-V).

\section{References}

Arora, R. (2005). Similarity solutions and evolution of weak discontinuities in a Van der Waals gas. The Canadian Applied Mathematics Quarterly, 13(4), 297-311.

Arora, R., \& Chauhan, A. (2019). Lie symmetry analysis and some exact solutions of (2+1)-dimensional KdV-Burgers equation. International Journal of Applied and Computational Mathematics, 5(1), 15.

Arora, R., \& Sharma, A. (2016). Similarity solutions of cylindrical shock waves in magnetogasdynamics with thermal radiation. Journal of Computational and Nonlinear Dynamics, 11(3), 031001.

Arora, R., Siddiqui, M.J., \& Singh, V. (2013). Similarity method for imploding strong shocks in a non-ideal relaxing gas. International Journal of Non-Linear Mechanics, 57, 1-9.

Bluman, G.W., \& Kumei. (1989). Symmetries and differential equations. Springer, New York, NY.

Chauhan, A., \& Arora, R. (2019). Self-similar solutions of cylindrical shock wave in a dusty gas. Indian Journal of Physics, 1-9.

Chauhan, A., Arora, R., \& Siddiqui, M.J. (2019). Propagation of blast waves in a non-ideal magnetogasdynamics. Symmetry, 11(4), 458.

Guderley, K. (1942). Starke kugelige und zylindrische verdichtungsstosse in der nahe des kugelmitterpunktes bnw. der zylinderachse. Luftfahrtforschung, 19, 302.

Hafner, P. (1988). Strong convergent shock waves near the center of convergence: a power series solution. SIAM Journal on Applied Mathematics, 48(6), 1244-1261.

Hydon, P.E. (2000). Symmetry methods for differential equations: A beginner's guide (Vol. 22). Cambridge University Press. 
International Journal of Mathematical, Engineering and Management Sciences

Vol. 4, No. 5, 1094-1107, 2019

https://dx.doi.org/10.33889/IJMEMS.2019.4.5-087

Logan, J.D. (1987). Applied mathematics: a contemporary approach. Wiley-Interscience Publication, John Wiley \& Sons, Inc., New York MR0907026 (88i:00020).

Nath, G., \& Singh, S. (2017). Flow behind magnetogas dynamic exponential shock wave in self-gravitating gas. International Journal of Non-Linear Mechanics, 88, 102-108.

Perry, R.W., \& Kantrowitz, A. (1951). The production and stability of converging shock waves. Journal of Applied Physics, 22(7), 878-886.

Rosenau, P., \& Frankenthal, S. (1976). Equatorial propagation of axisymmetric magnetohydrodynamic shocks. The Physics of Fluids, 19(12), 1889-1899.

Sharma, V., \& Arora, R. (2005). Similarity solutions for strong shocks in an ideal gas. Studies in Applied Mathematics, 114(4), 375-394.

Sharma, V., \& Radha, C. (1995). Similarity solutions for converging shocks in a relaxing gas. International Journal of Engineering Science, 33(4), 535-553.

Singh, J., \& Vishwakarma, P. (1983). Unsteady isothermal flow of a gas behind an exponential shock in magnetogasdynamics. Astrophysics and Space Science, 95(1), 111-116.

Singh, L.P, Husain, A., \& Singh, M. (2010). An analytical study of strong non-planar shock waves in magnetogasdynamics. Advances in Theoretical and Applied Mechanics, 3(6), 291-297.

Van Dyke, M., \& Guttmann, A. (1982). The converging shock wave from a spherical or cylindrical piston. Journal of Fluid Mechanics, 120, 451-462.

Vishwakarma, J.P, \& Nath, G. (2007). Similarity solutions for the flow behind an exponential shock in a non-ideal gas. Meccanica, 42(4), 331-339.

Whitham, G.B. (2011). Linear and nonlinear waves (Vol. 42). John Wiley \& Sons, New York.

Zeldovich, Y.B., \& Raizer, Y.P. (1965). Physics of shock waves and high-temperature hydrodynamic phenomena (Foreign Technology Div. Wright-Patterson AFB OH).

(c) (i) Original content of this work is copyright (C) International Journal of Mathematical, Engineering and Management Sciences. All rights reserved. Except of uses under a Creative Commons Attribution 4.0 International (CC BY 4.0) license at https://creativecommons.org/licenses/by/4.0/ 